\title{
Paolo Amaldi, Architecture, profondeur, mouvement
}

Gollion, Infolio, coll. « Projet et Théorie », 2012, 495 pages.

\section{Pierre Boudon}

\section{(2) OpenEdition \\ Journals}

Édition électronique

URL : http://journals.openedition.org/crau/513

DOI : $10.4000 /$ crau. 513

ISSN : 2547-5746

\section{Éditeur}

Éditions du patrimoine

Édition imprimée

Date de publication : 1 septembre 2013

Pagination : 94-102

ISBN : 978-2-7577-0109-6

ISSN : 1296-4077

\section{Référence électronique}

Pierre Boudon, « Paolo Amaldi, Architecture, profondeur, mouvement », Les Cahiers de la recherche architecturale et urbaine [En ligne], 28 | 2013, mis en ligne le 12 septembre 2017, consulté le 24 septembre 2020. URL : http://journals.openedition.org/crau/513 ; DOI : https://doi.org/10.4000/crau. 513 
Vattimo et François Lyotard discutent sur la valeur des pensées "fortes » et des pensées "faibles», en tant que réponses à la crise des grands récits de la modernité. Jacques Derrida et Peter Eisenman proposent une "résistance marginale ", où l'hédonisme individuel et l'expérimentation personnelle prennent la place du conflit historique. Jean Baudrillard et Jean Nouvel évoquent une " théorie hyperréaliste ", où la singularité, I'instant et l'urbanisation virtuelle façonnent la vie contemporaine. Rem Koolhaas élabore sa théorie de la Bigness et du Junkspace, qui sont les expressions des modes de vie de l'espace métropolitain indéfini.

Dans l'épilogue, le livre trace de nouvelles lignes de fuite pour une théorie de l'architecture. Pour sortir du paradigme moderne, la notion de "commun " - ce qui n'est pas privé, ni public - est proposée comme une des trajectoires possibles. En effet, l'origine du terme, le latin "munus », accueille l'idée du don à offrir aux autres, ce qui implique une réciprocité et une mutualité nouvelles. Contrairement aux logiques individualistes de la modernité, le " commun » renvoie à une richesse commune, autant matérielle (l'eau, la nature) que immatérielle (la société, les connaissances, les langages). Cela permettrait aussi d'établir des expressions de l'agir collectif (politique) et d'édifier des formes d'habitat (architecture / ville / territoire), véritablement ouvertes à l'espace et au temps du futur.
Ainsi, conçu par un « non-architecte », le livre a le mérite de proposer une relecture du rôle de l'architecture contemporaine, en travaillant sur une actualisation critique de ses termes et en confirmant sa responsabilité civile face à l'histoire et à la société. II reste peut-être une incertitude par rapport aux outils et aux formes concrètes d'action (aux architectes et aux politiciens de les trouver ?) et aux limites spatio-temporelles suggérées par l'auteur. Comme le théoricien Franco Berardi l'évoque dans la conclusion du livre, le cadre de travail de Marco Assennato est encore une fois la ville occidentale, ce qui pourrait limiter l'élan vers le futur soutenu par le texte. Une ouverture intéressante pourrait alors s'appuyer sur d'autres contextes spatiaux et sociaux, ainsi que sur d'autres croisements entre architecture, théorie et politique.

ROBERTA BORGHI

Architecte-urbaniste, enseignante à l'Énsa de Strasbourg et à l'université de Parme, membre de I'UMR Architecture, morphologie/morphogenèse urbaine et projet, Énsa et Insa de Strasbourg, et du laboratoire Architecture, musées, réseaux, université de Parme, département d'Ingénierie civile et architecture.

\section{Paolo Amaldi, Architecture, profondeur, mouvement}

Gollion, Infolio, coll. «Projet et Théorie », 2012, 495 pages.

L'ouvrage de Paolo Amaldi, Architecture Profondeur Mouvement, deuxième de la collection "Projet et Théorie », représente une somme non pas historique - bien qu'il emprunte le cours de l'histoire architecturale en Occident depuis le Quattrocento italien de Brunelleschi et Alberti jusqu'à Giedion et Le Corbusier mais, nous dirons, "épistémologique ». En effet, ce n'est pas tant l'enchaînement des "faits " comme événements stylistiques (œuvres remarquables, courants, périodes) qui importe que le fonds conceptuel sous-jacent à leur avènement. Ce n'est pas non plus un ouvrage qui emprunterait à une psychologie de la perception (comme chez Arnheim et ses références à la Gestalttheorie) une démarche cognitive appliquée à ces mêmes faits architecturaux, bien que par son titre et son point de départ on pourrait le supposer (par exemple, les références à Descartes, à Locke ou Berkeley, à Merleau-Ponty présent dès la préface, etc.). En fait, ceux-ci sont l'amorce et les points de référence d'une problématique caractérisée comme analyse épistémologique proprement architecturale de l'espace où ces notions (historiques, psychologiques) sont pour nous l'enjeu. 
Plus que d'architectures comme « objets » de ces analyses, nous avons affaire à une " scène » où ceux-ci exprimeraient la manifestation de dispositifs sous-jacents à titre de montages spéculaires (voir $\mathrm{Ce}$ que nous voyons, ce qui nous regarde, selon la belle expression de Georges Didi-Huberman).

Cette somme épistémologique s'échelonne en dix parties : " 01 Tout voir pour comprendre ", ou la mise en place d'un regard spéculatif issu de la tradition philosophique occidentale; " $02 \mathrm{La}$ ligne, présence réelle », en tant qu'architectonique de la perspective linéaire ; " 03 Clarté et description », en tant que déploiement historique et totalisant de cette opération spéculaire; " 04 Lignes, indices et graphes ", en tant qu'instrumentalisation de la ligne comme qualités naturelles (caractères, traits, aspects) ; " 05 L'épaisseur du vide », en tant que formation en creux travaillant ce dispositif, créatrice d'un écart entre un au-delà et un en-deça ; " 06 Par delà le regard », ou l'esthesis en réaction à toute forme de conceptualité délimitative ; « 07 Conscience, subconscience », en tant que lieu de cette transformation souterraine ; " 08 Immersion-abstraction », nouvelle spatialité en tant que déconstruction des formes enveloppantes et apparition d'un réseau de structures réticulées ; " 09 L'espace : forme ou expérience ?", en tant qu'enjeu existentiel du Mouvement moderne. Enfin, nous situerons la dernière partie, « 10 Le théâtre postmo- derne de la vacuité ", comme résultante d'un démembrement d'un tel parcours entre une hypermodernité (technologique) et une prémodernité (écologique). Ainsi la perspective (à point central) au Quattrocento est-elle au départ le lieu d'une élaboration spéculative révélée dans ce qu'on a appelé les « représentations urbinates » de la ville idéale (p. 53, 61), dues à l'entourage de Laurana, représentations dans lesquelles nous avons une confrontation entre des espaces de type concave (façades de palais de part et d'autre) et de type convexe (le macellum situé au centre) produisant cet effet d'engouffrement du regard de l'observateur dans la représentation. Or le même dispositif se retrouve à titre d'architecture (inachevée) dans la façade du Temple Malatesta à Rimini due à Alberti. C'est par rapport à cette première forme de montage exemplaire que I'on peut caractériser d'autres formes divergentes telles que le montage anti-axial, dérobant le point de vue unique, dans le Santo Spirito de Brunelleschi (p. 91-93), ou encore, le basculement d'une profondeur horizontale en une élévation verticale dans le Tempietto de San Pietro in Montorio dû à Bramante (p. 103-108), faisant disparaître toute assignation de point de vue à hauteur d'homme (il faut ainsi rétablir imaginairement la forme $d^{\prime}$ '« écrin » du cloître abbatial qui devait former l'entourage de ce tempietto).

Plus tard, à l'âge classique français (p. 136-141), nous avons affaire au déploiement d'un montage spéculaire selon le même regard dominant, non plus associé à une architecture proprement dite (l'église, le palais) mais à un territoire relevant de la même mise en scène (les jardins à Versailles, mettant en correspondance pièces d'eau et ciel, surfaces gazonnées et bosquets, le jeu des différentes terrasses d'où émerge la sculpture en tant que ponctuation rythmique des parcours). Or, exprimer dans le même plan le proche (les parterres, les pièces d'eau) et le lointain (les masses d'arbres, les étendues d'eau), le fini et I'infini à l'horizon, suppose l'introduction subreptice de motifs anamorphiques qui permettent d'accorder les uns avec les autres, de restituer une vraisemblance visuelle à l'ensemble à titre de lisibilité.

Avec les "jardins à la française ", nous sommes encore dans le domaine d'une territorialité étendue, même si ses confins (en termes d'horizon indéfini) préoccupent le théoricien (architecte ou géomètre). C'est au cours du xviiie siècle par contre que s'opère une rupture entre ce qu'on pourrait intituler le " paradigme " classique de la perspective issu de la Renaissance, maintenu comme forme de cadrage optique dans ses variantes, et l'émergence d'un " paysage " comme éclatement de ce dispositif d'encadrement ${ }^{1}$; rupture dans laquelle on pourrait parler d'une inversion des rapports dedans/dehors faisant apparaître les " éléments naturels » (fluides en tant que cours d'eau ou

1. Toutefois, cette réintroduction des conditions atmosphériques d'une perception - la notion d'un " espace-milieu », avant d'être un " espace-substance » - exige un retour sur la figure de Léonard de Vinci qui a été par anticipation le contradicteur d'Alberti ; d'où sa présence au début de la cinquième partie (p. 177-180) quant à une prospettiva di colore associée aux effets de profondeur. 
étendues marines, aériens en tant que nuées, brouillards, ou terrestres en tant que masses montagneuses; enfin le feu en tant que source d'énergie) comme fondamentaux de la représentation. La qualité de ce qui est mis en valeur comme montage de figures est ainsi transférée aux substrats qui les composent. On peut ainsi parler de " milieux " naturels (aérien, aquatique ou végétaux tel que la forêt) à la place d'ensembles composés.

Dans le domaine de l'architecture, on assiste au même déplacement de figures fermées à des figures ouvertes dont l'exemple type peut être celui des "places royales " (encadrant la statue du souverain) à la place Louis xv (la future place de la Concorde); mieux encore, aux places extérieures à la ville qui se prêtent aux fêtes de la Nation comme celle qui eut lieu sur le champ de Mars pendant la Révolution française (p. 246-247) ; enfin, jusqu'au projet d'Antolini pour le Foro Bonaparte à Milan (p. 256-262) qui représente l'apogée d'un tel déploiement spatial, soit d'espacement démesuré à titre de figure de l'évidement d'un tissu urbain. Cette transformation d'une figure fermée en une figure ouverte aura une longue histoire puisqu'on la retrouvera au $x x^{e}$ siècle dans celle entre l'îlot urbain compact et la forme démembrée des barres et des tours d'habitation du Mouvement moderne où la même notion d'évidement joue un grand rôle (justifié par des raisons d'hygiène, de circulation). Enfin, figure symétrique et inverse, nous aurions l'espace de renfermement, à la fois fermé et transparent de part en part, du panopticon qui a été pour Michel Foucault l'exemple révélateur de la notion de dispositif en tant que " machinerie visuelle » où le prisonnier n'est pas seulement enfermé mais scruté, soumis au regard omnipotent (nouvelle figure d'Argos) d'un gardien situé au centre de ce dispositif (p. 249) : "Le surveillant qui occupe le centre du système est un individu quelconque. II n'incarne aucun pouvoir suprême. II est partie prenante d'un dispositif (terme employé par Foucault) dans lequel le rôle de voyant et celui de voyeur peuvent se trouver renversés à tout moment. Plus encore, le système panoptique inaugure une nouvelle forme de regard dilué. Dans le panoptique, l'inspecteur surveille le prisonnier tout comme les gardiens. Chacun regarde l'autre tout en se sentant surveillé. Le gardien observe les détenus qui s'observent entre eux, dans la mesure où ils sont regroupés pour mieux s'autoéduquer. L'inspecteur est regardé par le peuple, qui peut à tout moment demander à voir les détenus ou contrôler les conditions de travail des gardiens ».

Le même dispositif gérera la prison, l'hôpital, la manufacture, à la fois pour des raisons disciplinaires et économiques.

Parallèlement à cette transformation, nous avons le passage d'une hétérogénéité constitutive des rapports entre la nature et la culture à une homogénéité des "éléments ", qu'ils soient naturels ou sociaux. La société n'est plus répartie en ordres, corporations ou statuts, mais en individus segmentés dont la notion de subjectivité " traverse "les différentes catégories (les écrits de Rousseau auront été l'un des propagateurs d'une telle individualité subjective) ${ }^{2}$. Là encore, nous avons une dissociation des individus formant une homogénéité de rapports au sens de leur uniformisation (par simplification, par réduction des particularismes).

Dans le domaine de la composition architecturale, cela n'est pas sans faire penser à la démarche combinatoire de JeanNicolas-Louis Durand où le " bâtiment " prend place dans une "économie " des formes génériques qui les associe les uns aux autres pour constituer, par permutation des pièces, des "types" socioéconomiques d'architecture (maisons, marchés, temples, portiques, hôpitaux, prisons) dénués d'effets stylistiques particuliers. Nous avons affaire à un catalogue de bâtiments civils, réglés sur une trame universelle (l'usage du papier quadrillé) et « horizontalisés » par couches superposées (p. 172-175). Tout ceci répond à une lisibilité parfaite de l'œuvre, à une positivité didactique de sa présence. Résumons cette longue évolution au cours du passage entre le $x v I^{e}$ et le $\mathrm{XIX}$ siècle.

La perte des points de vue (dans une déambulation aléatoire), la perte des cadrages (dans une dissolution des figures devenant des masses) engendrent
2. Par exemple, le théâtre en tant que lieu de rassemblement de la société subit cette transformation d'une hétérogénéité à une homogénéité avec les projets de Peyre et de De Wailly pour l'Odéon (p. 238-240), suivis, peu après, par celui de Besançon par Ledoux (p. 249-253) où la salle redevient un amphithéâtre "à la grecque » (comme celui de Palladio à Vicence), libéré de ses loges qui cloisonnaient l'assistance. Le théâtre devient ainsi le symbole d'un rassemblement civique. 
ainsi un sentiment d'immersion des sujets dans un " milieu-substance »; soit, défaisant le rapport disjonctif entre la nature et la culture où l'humain n'est plus qu'un appendice de l'ordre naturel, brassé par les éléments qui le submergent. Art des paysages, expressions picturales (notamment aquarellées avec Turner) et architectures finissent par se confondre dans une "vision » mystique des origines (les cathédrales-promontoires de Friedrich ou de Schinkel) se substituant à l'ancien cosmos étagé entre ciel et terre ; la "nature sauvage " se déploie en trois tropes, comme les intitule Amaldi (p. 307) : la montagne en tant cimes inaccessibles, la mer en tant qu'immensité (mais comportant des ailleurs éloignés et merveilleux), la grande forêt (avec Laugier, en particulier, assimilant l'ordre urbain et l'ordre naturel, le parc avec ses allées forestières devenant le lieu de leur rencontre interdéfinitionnelle).

Ainsi l'expressionnisme architectural allemand (avec Poelzig, les frères Luckhardt, Bruno Taut) est-il I'héritier de cette tradition romantique (tant littéraire que picturale ou architecturale) qui court tout au long du siècle jusqu'à la veille de la Première Guerre mondiale. Par exemple, dans l'expressionisme de Taut (voir Alpine Architektur, 1919, p. 309), nous avons une dissolution des cadres volumiques habituels de l'architecture en tant qu'opération de "transsubstantiation " par la lumière puisque la cité, transportée sur les cimes des Alpes, est transformée en un organisme cristallin illuminé par le soleil à travers une kaléidoscopie " en tant que dispositif déstructurant l'espace » (p. 311), à l'envers d'une perspective focalisante. La cité terrestre devient une cité céleste (ce qu'on retrouvera plus tard chez un Buckminster Fuller). Au Festspielhaus de Hellerau dessiné par Tessenow, le siècle retrouve le thème symbolique et mystique de la cathédrale gothique, baignée de lumière, mais aussi, intégrant la musique, la danse, la mise en scène (mais une mise en scène rompant avec la dramaturgie traditionnelle telle qu'elle était maintenue à Bayreuth dans les opéras de Wagner).

Paolo Amaldi accorde une grande importance à tout ce courant allemand, fréquenté par les intellectuels qui feront le siècle suivant (Jacques-Dalcroze, Appia, Mies van der Rohe, Gropius, le futur Le Corbusier) ${ }^{3}$. Nous avons ainsi la formation d'un Gesamtkunstwerk (œuvre d'art total, ou encore, "synthèse des arts ») intégrant, architecture, peinture, scénographie, danse, décliné sur un mode majeur à Hellerau et Bayreuth, ou sur un mode mineur (plus participatif que contemplatif) avec l'émergence des spectacles plus populaires qui attiraient les foules (dioramas, panoramas) comme immersion dans l'œuvre par captation physique du regard (et non plus seulement intellectuelle), par fascination des spectateurs. Pour Benjamin qui sera au $x x^{e}$ siècle le grand théoricien de cette " présence urbaine » enveloppante, tous ces arts mineurs constituent un milieu ambiant que les " passages » (à Paris, à Bruxelles, à Milan) cristallisent, fusionnant en une même activité commerce et distraction (le thème du flâneur dans la ville). Mais c'est aussi l'époque où apparaissent les notions de "goût du grand public ", d'" opinion de l'homme de la rue ", notions floues qui exprimèrent au $X X^{e}$ siècle un divorce de plus en plus grand entre une élite artistique sensible aux expériences modernes et la population dans son ensemble plus sensible aux ersatz de la grande culture passée.

Revenons sur l'intitulé général de cet ouvrage Architecture, profondeur, mouvement : à la notion d'architecture correspond celle d'une architectonique, " encadrée " par la perspective linéaire (faisceau de droites concourantes) depuis sa mise en forme canonique par Brunelleschi et Alberti en tant que dispositif spéculaire, et "encadrante » en ce qu'elle découpe une mise en scène, un paysage faisant monde; à la notion de profondeur correspond les effets suscités par cette mise en scène, à la fois comment elle organise un rapport entre fini et infini (rôle de l'horizon, des points de fuite) du côté de la scène, comment l'approfondissement de ce rapport finit par dissiper toutes espèces de contour (dans le rôle du sfumato, par exemple), et du côté du sujet observateur, comment elle " ouvre » un monde à la subjectivité du regard comme Einfuhlung (empathie)
3. Par différents aspects, l'école d'Hellerau anticipe ce que sera le programme du Bauhaus après la Première Guerre mondiale, notamment la pluralité des arts, cultivés simultanément, et l'ascèse des formes (par exemple, les scénographies d'Appia accompagnées de leurs jeux de lumière) ; le théâtre total de Gropius (1922) pour le scénographe Piscator participe d'un tel mouvement. 
comme mode sensible d'une intériorité en résonance avec un paysage (lequel pourra s'ouvrir à une vastitude). Enfin, avec le troisième terme : la notion de mouvement, nous avons l'apparition d'une dynamique, simultanée aux recherches contemporaines en physique (définition de la notion d'énergie avec Carnot, analyse de la lumière avec Young et Fresnel), qui prend en charge les dispositions préalables (architectonique en tant que réseau de lignes entrecroisées, profondeur en tant que dissolution des formes figées) pour les « mettre en mouvement " en tant que formes dynamiques (en particulier, grâce à la mise au point des modes d'enregistrement tels que la photographie, le chronophotographe de Marey, p. 366, ou le zoopraxiscope de Muybridge, p. 367), introduisant ainsi l'idée de flux, de continuité sérielle, de changement morphologique, de parcours (et de vitesse) en tant que rapport entre temps et espaces parcourus. D'un côté, la temporalité associée à tous ces phénomènes, devient fondamentale et en philosophie on voit apparaitre des théories du temps comme écoulement (Bergson, Husserl) ; de l'autre, aux formes d'un cadrage établi fait place la notion d'un champ visuel indéfinie où l'attention n'est plus celle d'un regard focalisé mais une attention flottante parcourant le champ comme balayage. Du regard encadré on passe ainsi à un regard décadré, atopique, au sens où il n'est plus focalisé sur des objets précis.
Auparavant, nous avons vu que la notion d'apprentissage des arts se dissolvait dans l'interrelation entre genres esthétiques (cas de l'école d'Hellerau où danse, mise en scène, gymnastique, musique, se fondaient dans une pratique globale; expérience que l'on retrouvera au Bauhaus) ; le "corps " n'est plus figé selon des canons préétablis mais " mobilisé » en tant que mouvements et gestes, créant une spatio-temporalité dynamique. L'œuvre d'art n'est plus une contemplation mise à distance mais la production d'un système d'effets (le corps peut exprimer une énergie, un effort tendu vers un but; bref, il devient une activité permanente).

Que ce soit dans la vie courante ou sur scène, ce corps est "déshabillé » au sens où il n'est plus le support d'une multitude d'empiècements qui le masque ; Loos en fera une éthique, du comportement comme de sa mise en scène.

Les "pratiques du corps » (esthétiques) sont un bon modèle de ce qui se passe pour les autres formes de la représentation (qu'elles soient architecturales ou picturales) ${ }^{4}$. Les volumes architecturaux se simplifient dans leur apparence (masses épurées, volumes d'épannelage), leur cadrage se diffracte par démultiplication jouant sur une sérialité, soit comme éclatement des entités compactes (cas du futurisme, p. 347), soit comme fragmentation par facettes des plans uniformes (cas de l'élémentarisme de De Stijl, p. 341) ; dans chacun

4. La place d'un lieu d'exercice du corps (gymnastique, détente) devient essentielle dans la définition du projet architectural; ainsi le solarium qui " coiffe » le sommet de la Villa Savoye de Le Corbusier. de ces cas, nous avons l'abolition de la notion de façade qui fut l'emblème (chez Alberti, par exemple) d'une perspective frontale.

Nous avons mentionné l'apparition des instruments de reproduction (photographie, chronophotographe et plus tard cinématographe) ; ceux-ci viennent, d'abord, " dédoubler " la notion de réalité en ce que ces appareils restituent exactement les apparences de ce qui est capté. Mais à cette première forme de présence qui n'agit que comme témoignage succédera une seconde phase où cette présence seconde vient interférer avec la première (dans le mode même de captation des scènes, leur façon « immersive " de les cadrer comme chez les peintres Caillebotte ou Degas) ; enfin, en tant que mode d'illustration d'ouvrages, cette présence seconde finira par s'imposer comme "index » de ce qu'il faut voir (effets de plongée et de contre-plongée surprenantes), comme " grille » permettant de déchiffrer les phénomènes observés. Cette lecture a pour effet de "brouiller " les rapports entre observation et fiction, de "valoriser » ce que le photographe veut signifier; cet avènement d'un cadrage photographique trouvera son apogée au Bauhaus où il prend place au rang d'art (avec Moholy-Nagy, Marianne Brandt) au même titre que la peinture ou l'architecture. L'emblème de cette esthétique ne sera plus la façade ou la perspective mais les vues plongeantes (ou contre- 
plongeantes) à travers les structures entrelacées de la Tour Eiffel, des ponts transbordeurs ou bien sûr des « transparences " architecturales du bâtiment luimême de Gropius (p. 358).

Ces mêmes moyens de reproduction permettent également de renouer avec la notion de parcours sériel et aléatoire, développé au XVIII ${ }^{e}$ siècle à propos des promenades dans les parcs. La première manifestation en est, peut-être, la description que Choisy a donnée de la montée à l'Acropole (p. 374 sq.) et qu'il a justement appelée "pittoresque " dans son Histoire de l'architecture ; cette description est célèbre ${ }^{5}$ car elle va devenir avec Le Corbusier l'icône de la « promenade architecturale ", thème dominant et récurrent (de la Villa Savoye en 1929 au Carpenter Center for the Visual Arts en 1964) dans son œuvre. Ainsi, l'architecture est-elle traversée comme paysage de même qu'elle est le lieu d'observation d'un paysage environnant puisque la promenade permet une contemplation active, nécessitant un effort (elle est toujours ascendante).

Nous venons d'insister sur les moyens de la reproduction technique (photographie et cinématographie) qui agissent comme composantes de l'œuvre et qui réïfient en quelque sorte la puissance du regard en le démultipliant par vues successives et/ou simultanées. Par exemple, chez Giedion (p. 385 sq.) dans son ouvrage Espace, temps, architecture (1941, première édition) qui deviendra la

5. Elle associe en fait une pluralité de thèmes déjà abordés : à la fois ses réminiscences à la déambulation dans un parc, celle d'un champ de perception temporalisé qui découvre au fur et à mesure les bâtiments de part et d'autre et dont l'échelonnement dans le texte correspond à autant d'instantanés " croqués » (quatre, pour être précis); enfin son association à un relevé archéologique minutieux fait au début de la carrière de l'historien.
Bible du Mouvement moderne, le bâtiment du Bauhaus de Gropius va devenir l'exemple princeps d'une telle économie des regards puisque, vu d'en haut (vue zénithale), vu tout autour (circulairement), vu par angles (rapprochés), vu entre les façades s'interpénétrant, etc., celui-ci devient un prisme oculaire que ce soit comme " mouvement » du regard l'enveloppant ou que ce soit comme " manipulation " de l'objet qu'on fait " miroiter " pour en dégager les différentes facettes. C'est ce regard "photographique " lié (regard cadré, regard en déplacement) qui impose ses règles à l'architecture et le même phénomène se retrouve chez Le Corbusier dans ses " promenades architecturales » où le bâtiment est découpé en vues particulières, ou inversement, dans ses grands panoramas (Plan Voisin, Ville pour trois millions d'habitants, où l'objectif est agrandi à la dimension d'un territoire. Comme au Quattrocento ${ }^{6}$, I'œil domine sur l'appréhension tactile (les écrits de Le Corbusier sont explicites à cet égard), mais en même temps, il est contraint par la diffraction du plan de représentation comme déroulement de vues successives; d'où le dilemme inscrit dans l'intitulé de cette neuvième partie: "l'espace : forme ou expérience ?" Forme comme entité synthétique ou expérience comme mouvement incessant du regard conduit par la marche? À la lecture de l'ouvrage de Paolo Amaldi, nous avons vraiment l'impression que la trajectoire de Le Corbusier a exprimé les

6. Ce retour peut être signifié par le mouvement qu'il lance avec Ozenfant, Le purisme (1921) faisant suite à Après le cubisme (1918) en tant que volonté d'un dépassement; c'est un retour à la théorie du disegno par redressement des profils, là où le cubisme finissait par émietter les volumes: "L'idée de forme précède celle de couleur. La forme est prééminente, la couleur n'est qu'un de ses accessoires », (Après le cubisme, p. 84). différents enjeux du champ architectural au $x x^{e}$ siècle, comme si sa quête d'une forme, d'une présence, d'un remodellement du monde, avait marqué notre discipline. Ainsi, si la période dite des "maisons blanches " (associée à ses recherches picturales et exprimée par l'aphorisme : " une maison, un palais ») a symbolisé le purisme, celle après la Seconde Guerre mondiale où ses préoccupations se déplacent vers la sculpture polychrome (symbole d'un art primitif, comme dans les groupes Ozon, Ubu, Totem... et dans le thème de la "Main ouverte » pour Chandigarh) a été marquée par ce que Reyner Banham appellera le Brutalisme dont l'Unité d'habitation de Marseille ou les Maisons Jaoul, parmi d'autres, sont des symboles marquants. On peut alors parler d'un déplacement d'une conception visuelle (du champ de perception) à une conception tactile où le "grain » de l'enveloppe devient prégnant (la texture, les matières), comme l'objet sonore va le devenir par rapport à l'harmonie classique (rappelons que la mère et le frère de Le Corbusier étaient musiciens et que des appareils de captation des sons, dans l'illustration de ses textes, vont faire leur apparition dans les années 1930)7.

De ce point de vue, le "cabanon " de Roquebrune-Cap-Martin (1952) occupe une position singulière : s'agitil encore d'" architecture " et de son ancêtre mythique, la « cabane primitive " (chez Vitruve, chez Laugier), ou bien d'un renoncement à modeler une

7. Sculptures polychromes, appareils de capture du son, notion d'horizons sonores perçus par l'oreille se retrouveront dans des œuvres complexes telles que Notre-Damedu-Haut à Ronchamp ou le Pavillon Philips à l'Exposition universelle de Bruxelles en 1958 
forme qui n'est pas sans évoquer ce que sera quelques années plus tard le non design d'un Moore par rapport au total design (Fuller, mais aussi les divers courants italiens, No-Stop City, Superstudio ou Archizoom Associati ? Comme l'écrit Amaldi (p. 414-415) : «En revenant aux années de contestation du langage international, nous observons que les questions de l'existence, de l'identité et de l'appartenance sous-tendent l'idée du lieu, que résume la célèbre expression de Peter Smithson, prononcée lors du $10^{\mathrm{e}} \mathrm{CIAM}$ en 1956 : " se sentir comme quelqu'un qui vit quelque part ». Et d'ailleurs, si le terme " espace » a largement marqué la théorie de la période d'avantguerre et se définit selon une modalité de perception, on observe que ce terme sera progressivement substitué dans la période d'après-guerre par ceux de lieu ou de contexte, chargés, tous deux, $d^{\prime}$ une dimension psychologique, d'un vécu et d'une valeur d'usage, et investis sémantiquement par un réseau de références convoquées in absentia ( $d$ 'où son épaisseur intertextuelle), empruntant leurs instruments aux sciences cognitives, aux théories de l'information, mais aussi à la topologie. La notion de contexte renvoie enfin à une localisation de l'architecture, à un ancrage sur un site qui est censé lui transférer une partie de sa substance. »

Il y a donc un enjeu existentiel (contemporain de certaines philosophies) et c'est pourquoi nous pouvons parler d'un dilemme entre forme et expérience; la première se fond-elle dans la seconde, ou réciproquement, la seconde se modèle-t-elle sur la première ? Le thème d'une expérience vécue, située, est celui d'une perception du monde; mais c'est aussi, parallèlement celui d'une tradition en tant que vécu collectif et c'est pourquoi nous avons vu réapparaître avec force des thèmes vernaculaires dans certains courants postmodernes, symbolisés par exemple par les analyses de Christian Norberg-Schulz dont le parcours intellectuel récapitule bien ce passage d'une psychologie des formes perceptives (son premier ouvrage) à un retour aux traditions vernaculaires ${ }^{8}$ sous l'égide de Heidegger en tant que continuité symbolique; sous cet aspect, le Mouvement moderne constitue évidemment une réduction drastique, " une atrophie symbolique, idée partagée dans les mêmes années par Rob Krier et la mouvance postmoderniste » (p. 416). C'est donc le thème d'une identité culturelle, d'un habiter communautaire, qui devient l'épicentre d'une problématique architecturale et celle-ci se retrouvera par exemple dans le courant des "Team $X$ » (le couple des Smithson, Aldo van Eyck, Giancarlo de Carlo, parmi d'autres) qui représente un regard critique sur cette Modernité (plus exactement sur le Style International avec lequel on finit par la confondre).

L'œuvre de Le Corbusier, pendant les années 1950, devient déroutante aux yeux des théoriciens du Mouvement moderne (Giedion, Hitchcock, Argan) ou des jeunes architectes (Stirling, Kahn) engagés dans sa continuation, en ce qu'elle remet en question par sa pratique esthétique même les principes sur lesquels cette Modernité s'était constituée (notamment, la rationalité orthogonale et le principe fonctionnaliste dont était issue la Charte d'Athènes). La chapelle de Ronchamp en est l'exemple frappant mais aussi le couvent de la Tourette, sans parler des derniers projets tels que l'hôpital de Venise ou l'église de Firminy terminée trente ans plus tard.

Dans tous ces cas on peut parler d'une complexité de l'œuvre, au sens pro pre, qui ne peut être réduite à des principes simples d'explication ; I'œuvre exprime un nœud de relations et c'est dans cet esprit que l'on peut suivre l'analyse remarquable que Paolo Amaldi (p. 423-432) fait du couvent de la Tourette, "œuvre duale » comme il l'écrit (p. 423), en ce qu'elle est partagée par deux principes opposés : une perception visuelle et mesurable de l'ensemble qui décline l'œuvre en ses différentes parties conventionnelles (la porterie, les cellules, le réfectoire, l'église, le clocher), exposées sous la lumière, et une perception tactile, plus secrète, associée à la pénombre et que l'on peut situer entre l'oratorio (caché au cœur de l'édifice puisqu'on ne le perçoit pas de l'extérieur) et la crypte comme partie la plus « profonde » de l'édifice, associée

8. Et, pour certains, académiques (voir I'engouement pour le Style Beaux-Arts, I'exposition d'Arthur Drexler, The Architecture of the École des Beaux-Arts, au Moma de New York en 1975). 
au sol terrestre sur lequel elle repose. Il y a donc deux lectures, l'une conventionnelle, participant du schéma traditionnel du couvent, l'autre plus secrète en ce qu'elle compose ces deux modes de perception (dans l'oratorio, dans la crypte) où ils forment une association intime (antithétique, non-réversible entre le dedans et le dehors). Pour Le Corbusier, cette réalisation exprimait ce qu'il entendait par l' " espace indicible » (ou incommensurable, comme l'était la notion de "sublime " au xviiie siècle) qui constituerait le cœur de l'expérience architecturale?

La dernière partie de l'ouvrage, « le théâtre postmoderne de la vacuité » - avec ses deux expressions fortes, " théâtre " et " vacuité ", laissant entendre une "fin de partie " comme dans le théâtre de l'absurde de Beckett - comporte donc une part d'ambiguïté. D'une part, on peut penser à une forme de désenchantement qui suit la période grandiose de la Modernité (c'est la lecture rétrospective que l'on peut faire de I'histoire de l'architecture); d'autre part, il s'agit aussi d'aborder l'extrême richesse de nombreux courants d'analyse qui ont participé de l'élargissement de cette même notion de Modernité ${ }^{10}$.

On pourrait donc parler de cette dernière comme établissement d'une méta-architecture (mais l'expression n'apparaît pas dans l'ouvrage) en tant que design total définissant les trois instances fondatrices : a) principes générateurs de la forme (design thinking) ; b) notion de contrôle

9. Cette notion d'un " espace indicible » a été définie dans un numéro hors-série de l'Architecture d'aujourd'hui en 1946. À propos de cette œuvre, on pourrait également parler d'une "clarté labyrinthique », expression due à Aldo van Eyck.

10. II faut distinguer ces deux expressions : "postmoderne » et " postmodernisme », I'une caractérisant une période qui fait suite à la précédente à travers une " crise d'identité » de cette Modernité (c'est, cybernétique, psychologique ou bioclimatique (ainsi la notion $d^{\prime}$ '" environnement " se substitue à celle $d^{\prime}$ " espace » ou de «lieu ») ; c) enfin, notion d'échelle territoriale dont on déduira des formes locales; ce seront les différentes études sur la notion de "paysage urbain ", de " vision en mouvement à partir de l'autoroute ", de "lisibilité " de la forme urbaine dans sa disparité.

Ainsi, si le début de cette dernière partie s'ouvre sur la figure quasi-légendaire de Mies van der Rohe, figée dans la forme hiératique de ses immeubles américains (à New York, à Chicago, à Toronto) dont l'image inaltérable peut être reproduite à l'infini comme dans un jeu de miroirs parallèles et dont les disciples ne peuvent que répéter la formule, par contre, elle laisse la place à une richesse d'analyses où s'entrecroisent des recherches issues de la Gestalttheorie (notamment au MIT, avec les recherches de Györgi Kepes et de Kevin Lynch, p. 437, p. 440-441), d'une remise en question de la Modernité pour la dépasser (expériences du Team $X$ dues aux Smithson, à Aldo van Eyck ou à Woods et Candilis à Berlin), ou encore (p. 441), pour l'extrapoler dans les expériences sur les mégastructures (les Métabolistes japonais, Kenzo Tange dans la baie de Tokyo, mais aussi le Groupe d'étude d'architecture mobile (geam) à Paris avec Yona Friedman - projets dans lesquels les recherches sur les structures technologiques de grande dimension, Fuller, Wachsmann,

par exemple, le Team $X$ qui représente un tel mouvement de réflexion critique), I'autre se caractérisant en termes de style architectural et qui prend, avec plus ou moins d'arrogance, le contrepied de la précédente. Ainsi, à l'ordre moderne répond le désordre postmoderniste (vernaculaire, éclectique, ludique). On notera également que l'analyse s'arrête aux années 1980 et n'aborde pas la période plus récente où l'on voit renaître une autre forme de Modernité, plus agressive, plus
Le Ricolais, se mélangent avec une science-fiction, p. 445). C'est également la période d'une redécouverte des expériences constructivistes soviétiques des années 1930 (Leonidov, Chernikhov, El Lissitzky) sous le nom d'« architectures fantastiques" ".

À ce foisonnement relevant d'un design au sens large (technologique, écologique, plastique), on voit apparaître en opposition une critique sociopolitique qui n'est plus uniquement le fait d'analyses universitaires (sociologie ou psycho-sociologie urbaines) mais celui d'analyses engagées dans les mouvements sociaux contemporains pour défendre l'idée d' " espace public " ou encore de "droit à la ville " (avec Henri Lefebvre et plus tard avec Giancarlo de Carlo qui éditera une version italienne similaire à Sociologie \& société, p. 449 sq.). Ces analyses engagées n'ont pas pour but de réformer ou de proposer de nouvelles formules architecturales mais de les critiquer radicalement au nom d'une liberté intellectuelle ${ }^{11}$, de s'approprier l'espace urbain tel qu'il existe présentement (on retrouve dans cette tendance, à la fois, les analyses situationnistes de Guy Debord et de sa « psychogéographie de Paris », p. 454, et les analyses épistémologiques de Foucault sur le panopticon de Bentham).

L'historien et philosophe italien Manfredo Tafuri correspond sans doute à la jointure de ces deux versants d'une analyse critique de la Modernité, l'une

" scientiste » lorsqu'elle s'appuie sur des modélisations informatiques.

11. Ce courant, producteur de Fêtes de la musique et de théâtre dans la rue, n'est pas sans évoquer ce que furent les Fêtes Nationales lors de la Révolutions française, sur un mode toutefois plus ludique que politique. 
relevant des divers courants propres au design (dont la diversité peut être apparentée à une vacuité), l'autre relevant d'une analyse critique marxiste. Pour Tafuri, nous assistons à la disparition d'une Modernité architecturale (à sa production inventive), à la déshérence de ses formes tombant dans un " marché » capitaliste où l'on peut choisir ce que l'on veut (c'est le retour à une forme d'éclectisme propre au XIX ${ }^{\mathrm{e}}$ siècle). L'image postmoderniste de la Piazza d'Italia de Charles Moore (p. 459) à la Nouvelle-Orléans, qui clôt cette dernière partie, correspond bien à cet espace de la vacuité, fait de collages et qui, pour Tafuri, rappelle les gravures des Carceri de Piranèse, espace fantastique inconstructible mais saisissant pour le regard et qui n'est pas sans faire penser à ces villes du désert (telles le Las Vegas vanté par Robert Venturi et Denise Scott-Brown) dont le seul attrait est le jeu.

Certes, l'ouvrage de Paolo Amaldi ne pouvait couvrir la totalité de cette épopée qu'a été l'architecture occidentale depuis la première Renaissance avec Brunelleschi et Alberti. Ainsi peut-on regretter I'absence de certaines figures telles que celle de Loos ou de Terragni qui ont joué un rôle historique important, rétrospectivement mis en valeur (mais, rappelonsle, l'ouvrage n'est pas une anthologie des nombreux courants qui ont animé cette histoire mais un questionnement sur le sens de celle-ci). Encore une fois, ce que l'ouvrage souligne, c'est la continuité d'une problématique intellectuelle ouverte à la Renaissance (celle du regard, de sa mise en scène, de ses diffractions) indépendante d'une Tradition (au sens anthropologique) qui n'a fait que se perpétuer parallèlement dans ses formes classiques.

PIERRE BOUDON

Professeur honoraire, directeur de recherche associé au laboratoire d'Étude de l'Architecture Potentielle (LÉAP), université de Montréal et au laboratoire Langage, Logique, Informatique, Cognition (LALIC), université de Paris-Sorbonne. 